\title{
Gabor fusion master slave optical coherence tomography
}

Cernat, Ramona; Bradu, Adrian; Israelsen, Niels Møller; Bang, Ole; Rivet, Sylvain; Keane, Pearse A; Heath, David-Garway; Rajendram, Ranjan; Podoleanu, Adrian

Published in:

Biomedical Optics Express

Link to article, DOI:

10.1364/BOE.8.000813

Publication date:

2017

Document Version

Publisher's PDF, also known as Version of record

Link back to DTU Orbit

Citation (APA):

Cernat, R., Bradu, A., Israelsen, N. M., Bang, O., Rivet, S., Keane, P. A., Heath, D-G., Rajendram, R., \& Podoleanu, A. (2017). Gabor fusion master slave optical coherence tomography. Biomedical Optics Express, 8(2), 813-827. https://doi.org/10.1364/BOE.8.000813

\section{General rights}

Copyright and moral rights for the publications made accessible in the public portal are retained by the authors and/or other copyright owners and it is a condition of accessing publications that users recognise and abide by the legal requirements associated with these rights.

- Users may download and print one copy of any publication from the public portal for the purpose of private study or research.

- You may not further distribute the material or use it for any profit-making activity or commercial gain

- You may freely distribute the URL identifying the publication in the public portal 


\title{
Gabor fusion master slave optical coherence tomography
}

\author{
Ramona Cernat, ${ }^{1}$ Adrian Bradu, ${ }^{1,}{ }^{*}$ Niels Møller Israelsen, ${ }^{2}$ Ole \\ Bang, ${ }^{2}$ Sylvain Rivet, ${ }^{3}$ Pearse A. Keane, ${ }^{4}$ David-Garway Heath, ${ }^{4}$ \\ Ranjan Rajendram, ${ }^{4}$ and Adrian Podoleanu ${ }^{1}$ \\ ${ }^{I}$ Applied Optics Group, School of Physical Sciences, University of Kent, Canterbury CT2 7NH, Kent, \\ $U K$ \\ ${ }^{2}$ DTU Fotonik, Department of Photonics Engineering, Technical University of Denmark, DK-2800 \\ Kongens Lyngby, Denmark \\ ${ }^{3}$ Université de Bretagne Occidentale, EA 938 Laboratoire de Spectrométrie et Optique Laser, 6 Avenue \\ Le Gorgeu, C.S. 93837, 29238 Brest Cedex 3, France \\ ${ }^{4}$ NIHR Biomedical Research Centre at Moorfields Eye Hospital NHS Foundation Trust and UCL \\ Institute of Ophthalmology, London, EC1V 9EL UK \\ *a.bradu@kent.ac.uk
}

\begin{abstract}
This paper describes the application of the Gabor filtering protocol to a Master/Slave (MS) swept source optical coherence tomography (SS)-OCT system at $1300 \mathrm{~nm}$. The MS-OCT system delivers information from selected depths, a property that allows operation similar to that of a time domain OCT system, where dynamic focusing is possible. The Gabor filtering processing following collection of multiple data from different focus positions is different from that utilized by a conventional swept source OCT system using a Fast Fourier transform (FFT) to produce an A-scan. Instead of selecting the bright parts of Ascans for each focus position, to be placed in a final B-scan image (or in a final volume), and discarding the rest, the MS principle can be employed to advantageously deliver signal from the depths within each focus range only. The MS procedure is illustrated on creating volumes of data of constant transversal resolution from a cucumber and from an insect by repeating data acquisition for 4 different focus positions. In addition, advantage is taken from the tolerance to dispersion of the MS principle that allows automatic compensation for dispersion created by layers above the object of interest. By combining the two techniques, Gabor filtering and Master/Slave, a powerful imaging instrument is demonstrated. The master/slave technique allows simultaneous display of three categories of images in one frame: multiple depth en-face OCT images, two cross-sectional OCT images and a confocal like image obtained by averaging the en-face ones. We also demonstrate the superiority of MS-OCT over its FFT based counterpart when used with a Gabor filtering OCT instrument in terms of the speed of assembling the fused volume. For our case, we show that when more than 4 focus positions are required to produce the final volume, MS is faster than the conventional FFT based procedure.
\end{abstract}

(C) 2017 Optical Society of America

OCIS codes: (110.4500) Optical coherence tomography; (170.0180) Microscopy; (170.3890) Medical optics instrumentation; (180.6900) Three-dimensional microscopy; (170.0170) Medical optics and biotechnology; (100.0100) Image processing; (110.0110) Imaging systems.

\section{References and links}

1. D. Huang, E. A. Swanson, C. P. Lin, J. S. Schuman, W. G. Stinson, W. Chang, M. R. Hee, T. Flotte, K. Gregory, C. A. Puliafito, and J. G. Fujimoto, "Optical coherence tomography," Science 254(5035), 1178-1181 (1991).

2. W. Drexler and J. G. Fujimoto, Optical Coherence Tomography: Technology and Applications (Springer, 2015, Second edition).

3. A. G. Podoleanu and R. B. Rosen, "Combinations of techniques in imaging the retina with high resolution," Prog. Retin. Eye Res. 27(4), 464-499 (2008).

4. M. Wojtkowski, R. Leitgeb, A. Kowalczyk, T. Bajraszewski, and A. F. Fercher, "In vivo human retinal imaging by Fourier domain optical coherence tomography,” J. Biomed. Opt. 7(3), 457-463 (2002). 
5. S. R. Chinn, E. A. Swanson, and J. G. Fujimoto, "Optical coherence tomography using a frequency-tunable optical source," Opt. Lett. 22(5), 340-342 (1997).

6. M. Bonesi, M. P. Minneman, J. Ensher, B. Zabihian, H. Sattmann, P. Boschert, E. Hoover, R. A. Leitgeb, M. Crawford, and W. Drexler, "Akinetic all-semiconductor programmable swept-source at $1550 \mathrm{~nm}$ and $1310 \mathrm{~nm}$ with centimeters coherence length," Opt. Express 22(3), 2632-2655 (2014).

7. O. O. Ahsen, Y. K. Tao, B. M. Potsaid, Y. Sheikine, J. Jiang, I. Grulkowski, T.-H. Tsai, V. Jayaraman, M. F. Kraus, J. L. Connolly, J. Hornegger, A. Cable, and J. G. Fujimoto, "Swept source optical coherence microscopy using a $1310 \mathrm{~nm}$ VCSEL light source," Opt. Express 21(15), 18021-18033 (2013).

8. Z. Ding, H. Ren, Y. Zhao, J. S. Nelson, and Z. Chen, "High-resolution optical coherence tomography over a large depth range with an axicon lens," Opt. Lett. 27(4), 243-245 (2002).

9. R. A. Leitgeb, M. Villiger, A. H. Bachmann, L. Steinmann, and T. Lasser, "Extended focus depth for Fourier domain optical coherence microscopy," Opt. Lett. 31(16), 2450-2452 (2006).

10. D. Lorenser, C. Christian Singe, A. Curatolo, and D. D. Sampson, "Energy-efficient low-Fresnel-number Bessel beams and their application in optical coherence tomography," Opt. Lett. 39(3), 548-551 (2014).

11. A. Curatolo, P. R. Munro, D. Lorenser, P. Sreekumar, C. C. Singe, B. F. Kennedy, and D. D. Sampson, "Quantifying the influence of Bessel beams on image quality in optical coherence tomography," Sci. Rep. 6, 23483 (2016).

12. J. P. Rolland, P. Meemon, S. Murali, K. P. Thompson, and K. S. Lee, "Gabor-based fusion technique for Optical Coherence Microscopy," Opt. Express 18(4), 3632-3642 (2010).

13. V. J. Srinivasan, H. Radhakrishnan, J. Y. Jiang, S. Barry, and A. E. Cable, "Optical coherence microscopy for deep tissue imaging of the cerebral cortex with intrinsic contrast," Opt. Express 20(3), 2220-2239 (2012).

14. M. Hughes and A. G. Podoleanu, "Simplified dynamic focus method for time domain OCT," Electron. Lett. 45(12), 623-624 (2009).

15. A. G. Podoleanu and A. Bradu, "Master-slave interferometry for parallel spectral domain interferometry sensing and versatile 3D optical coherence tomography," Opt. Express 21(16), 19324-19338 (2013).

16. A. Bradu, K. Kapinchev, F. Barnes, and A. Podoleanu, "Master slave en-face OCT/SLO,” Biomed. Opt. Express 6(9), 3655-3669 (2015).

17. A. Bradu, K. Kapinchev, F. Barnes, and A. Podoleanu, "On the possibility of producing true real-time retinal cross-sectional images using a graphics processing unit enhanced master-slave optical coherence tomography system," J. Biomed. Opt. 20(7), 076008 (2015).

18. A. Bradu, S. Rivet, and A. Podoleanu, "Master/slave interferometry - ideal tool for coherence revival swept source optical coherence tomography," Biomed. Opt. Express 7(7), 2453-2468 (2016).

19. S. Rivet, M. Maria, A. Bradu, T. Feuchter, L. Leick, and A. Podoleanu, "Complex master slave interferometry," Opt. Express 24(3), 2885-2904 (2016).

20. Pacific Laser Equipment website: http://plequipment.com.

21. A. Bradu, M. Maria, and A. G. Podoleanu, "Demonstration of tolerance to dispersion of master/slave interferometry," Opt. Express 23(11), 14148-14161 (2015).

22. J. P. Su, Y. Li, M. Tang, L. Liu, A. D. Pechauer, D. Huang, and G. Liu, "Imaging the anterior eye with dynamicfocus swept-source optical coherence tomography," J. Biomed. Opt. 20(12), 126002 (2015).

23. C. Costa, A. Bradu, J. Rogers, P. Phelan, and A. Podoleanu, "Swept source optical coherence tomography Gabor fusion splicing technique for microscopy of thick samples using a deformable mirror," J. Biomed. Opt. 20(1), $016012(2015)$.

24. A. Bradu and A. G. Podoleanu, "Imaging the eye fundus with real-time en-face spectral domain optical coherence tomography," Biomed. Opt. Express 5(4), 1233-1249 (2014).

25. P. Meemon, J. Widjaja, and J. P. Rolland, "Spectral fusing Gabor domain optical coherence microscopy," Opt. Lett. 41(3), 508-511 (2016).

\section{Introduction}

Optical coherence tomography (OCT) [1,2] is a continuously evolving technology, providing high-axial resolution imaging. The beauty of the technology is that the axial (longitudinal) resolution in the image is decoupled from the transversal (lateral) resolution. The transversal resolution continues to be dependent on the interface optics in the sample arm of the interferometer, mainly on the numerical aperture of the microscope objective employed to focus light on the sample, as in confocal microscopy. The axial resolution however is determined by the bandwidth of the optical source employed. This has allowed achieving high axial resolution when imaging the retina in the living eye, despite the fact that retina is relatively far away from the eye lens. To this goal, optical sources with sufficient broad optical spectrum are used. This is one of the reasons why OCT became so popular in ophthalmology [3].

Spectrometer based [4] and swept source based OCT technologies [5] produce A-scans via a Fourier transform. All points in depth along the A-scan are produced under a single focus 
adjustment. Therefore, in the OCT practice, low numerical aperture (NA) interface optics is used to enable a confocal profile width or depth of focus (DOF), comparable with the axial range (AR) of the OCT. If good transversal resolution is needed along the axial depth, then by increasing the NA of the interface optics, the confocal gate width becomes narrower than the axial range. The problem presented is that the region of highest quality (as determined by contrast and resolution) is limited by the width and position of the DOF interval within the AR. It is also known that swept-source (OCT) instruments can provide longer axial imaging range than their camera based counterparts, exceeding several $\mathrm{cm}$ using akinetic [6] or tunable VCSEL (vertical-cavity surface-emitting lasers) [7] long coherence swept sources. Efforts have been made to extend the DOF whilst maintaining high lateral resolution. One approach uses an axicon lens to produce a Bessel beam. The DOF is enlarged at the expense of lowered sensitivity, making the method less suitable for imaging samples of low reflectance $[8,9]$, although progress has been made to improve its efficiency [10]. Very recently [11], for low numerical apertures and high scattering anisotropy, it was demonstrated that better contrast can be obtained over an extended depth range by a Gaussian beam combined with dynamic focusing. However, dynamic focus solution is restricted to time domain OCT only.

A solution suitable for high numerical aperture interface optics and applicable to spectral (Fourier) domain OCT was reported based on Gabor filtering [12,13], where acquisition was repeated for several focus positions, $R=A R / D O F$ that shifted the confocal gating profile incrementally through the sample. An OCT cross-sectional image was acquired at $\mathrm{R}=5$ different focus positions dictated by a liquid lens. From each of the 5 images, the in-focus region was extracted and then spliced together to form a single image wherein all regions are in focus. It was determined that 5 repetitions of data acquisition with shifted focus were sufficient as the DOF interval was approximately $1 / 5$ th of the AR.

In conventional FFT based swept source OCT, to perform Gabor filtering, the brightest parts of A-scans for each focus position are selected while the rest of A-scans are discarded. The selected parts of A-scans are fused into final B-scans or into a final volume of the tissue. In this way, in the final B-scan, or final volume, better transversal resolution along depth is achieved than when using a single focus position. In other words, for each spectral sweep, the FFT delivers more data than retained in the final image or final volume. In contrast, in time domain OCT, for each focus position, a single set of data is acquired, the set corresponding to the depth of interest selected by both gates.

In this paper a method is presented that although uses SS-OCT, can restrict the coherence gating to only the depths of interest, similarly to dynamic focusing [14] in time domain OCT. However, different from time domain, information from more than a single depth is acquired and delivered. Optimally, information is provided from the depths values within the DOF, as determined by the confocal gate of the interface optics. This operation can be repeated for each position of the focus.

The Master/Slave (MS) OCT method [15] is employed here, where the FFT is replaced with a comparison operation of the signal at the photodetector output with replicas of the same signal (masks) corresponding to the photo-detected signal obtained when a mirror is used at different axial positions [16-19]. For each depth position, different masks can be used. The procedure can replace the dynamic focus in time domain OCT, where in this case the set of masks is changed in synchronism with the focus shift. It is shown that the MS procedure allows real time display of three types of images in a single frame: several en-face, two Bscans and a compound en-face confocal image for guidance.

The paper presents the peculiarities of combining Gabor filtering (GF) with MS-OCT, incorporated into a GF/MS-OCT instrument. 


\section{Materials and methods}

\subsection{Optical coherence tomography imaging instrument}

The assembled SS-OCT instrument used here is schematically presented in Fig. 1. A swept source (Axsun Technologies, Billerica, MA), $1310 \mathrm{~nm}$ central wavelength, $12 \mathrm{~mm}$ coherence length, $106 \mathrm{~nm}$ FWHM bandwidth in the range $(1256.6 \mathrm{~nm}-1362.8 \mathrm{~nm})$ with an average output power of $18 \mathrm{~mW}$ and a scanning rate of $50 \mathrm{kHz}$ is used. Light from the SS is conveyed towards the sample and reference arm via the directional coupler $\mathrm{DC}_{1}, 20 \%$ of the power being directed towards the sample. In the sample arm, an achromatic lens $\mathrm{L}_{1}$ is supported by a miniature linear stage [20]. Signals back-scattered by the sample and from the reference arm are conveyed by the second directional coupler $\mathrm{DC}_{2}$ (50/50 splitting ratio) and interfere on the photodetectors of a balanced photo-detector unit, BPD (Thorlabs, Newton, New Jersey, Model PDB460C, $200 \mathrm{MHz}$ electronic bandwidth).

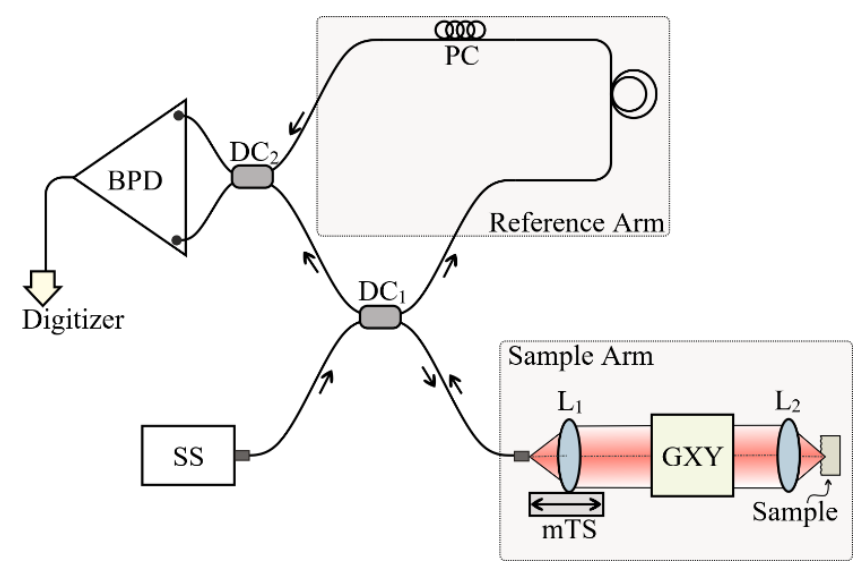

Fig. 1. The experimental set-up of the GF/MS-OCT imaging instrument. SS: swept source laser, $\mathrm{DC}_{1-2}$ : directional couplers, $\mathrm{L}_{1,2}$ : achromatic lenses, PC: polarization controller, mTS: miniature translation stage, GXY: pair of orthogonal galvo-scanners, BPD: balance photodetector.

The radio frequency signal at the output of the BPD is digitized using a 12-bit waveform digitizer (Alazartech, Quebec, Canada, model ATS9350 - $500 \mathrm{MS} / \mathrm{s}$ ). An "in-house" Master/Slave software, similar to the one used in [16] created in LabVIEW (National Instruments, Austin, Texas) is used to display three categories of images at each display refresh. Apart from the simultaneous display of 9 en-face OCT images, another feature presented here is the capability of assembly of a confocal lookalike image. The number of enface images, as well as the differential distance between them, is adjustable, which allows the generation of an adjustable thickness confocal image. The organization of the display is similar to that in [16], where the compute unified device architecture (CUDA) programing on a graphics processing unit (GPU) allowed real time presentation of three types of images. Two B-scan images are presented simultaneously with the display of several en-face OCT images and the confocal lookalike image. This is made possible by the MS method, as described in [16], which is applied by parallel processing of signals from the different depths of the lateral reflectivity profiles (T-scans) assembled together. As a distinction from [16], the same functionality is obtained here using the computer processing unit (CPU) only, based on our recent progress on the MS algorithm using manipulation of matrices [18, 19].

\subsection{Focus adjustment}

A miniature motorized miniature high-precision linear stage, as shown in Fig. 2(a) (model UMS-5 Laser Pacific Equipment, Tustin, CA, under R\&D) [20], with a total travel range of $6.5 \mathrm{~mm}$ corresponding to 76700 micro-steps, a minimum increment of $1 \mu \mathrm{m}$ and maximum 
speed of $1.5 \mathrm{~mm} / \mathrm{s}$ is used to shift an achromatic lens, $\mathrm{L}_{1}$, which gives 11800 micro-steps $/ \mathrm{mm}$ (11.8 micro-steps/micron), with 360 micro-steps per rotation. The size of the linear stage is 42 $\mathrm{mm}$ long, the height is $19 \mathrm{~mm}$, and the width is $20 \mathrm{~mm}$.

The miniature linear stage is controlled by a stepper motor controller (model SSP-21 Laser Pacific Equipment) with user selectable micro stepping values. We run the stage for 10-20 seconds and stop for 1-2 minutes and then run again, in order to avoid overheating the electronics. This is not an important impediment as acquisition of several 3D data sets in less than a second is followed by inspection, that is performed on the stored images.

The focusing lens $\left(\mathrm{L}_{1}\right)$ used in the object arm (Fig. 1) is an achromat doublet ( $\mathrm{f}=19 \mathrm{~mm}$, AC127-019-C Thorlabs), glued on a PolyLacticAcid (PLA) block holder created with a 3D printer. The scanning lens $\left(\mathrm{L}_{2}\right)$ contains two achromat doublets (the same model as the focusing lens $\mathrm{L}_{1}$ ) for enhanced transversal resolution. The PLA block holder [Fig. 2(b)] was specially created to adapt the height required by the optical fiber input and a low weight required by the miniature translation stage load specifications (maximum $0.25 \mathrm{~N}$ ).

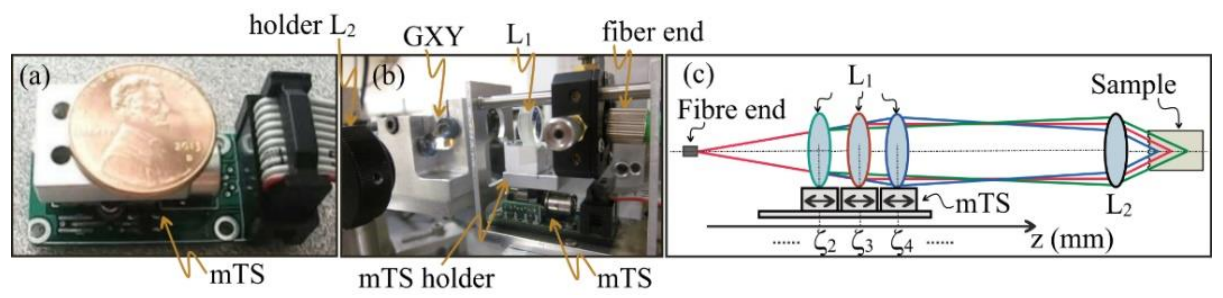

Fig. 2. (a) Photograph of the miniature linear stage used to move lens $L_{1}$. (b) Photograph of the miniature translation stage and its assembly close to the scanning head. (c): object arm ray tracing through $\mathrm{L}_{1}$ and $\mathrm{L}_{2}$ lenses when the mTS is in the collimating position $\left(\zeta_{3}=0\right)$ and for two un-collimated beam situations $\left(\zeta_{2}\right.$ and $\left.\zeta_{4}\right)$.

\subsection{Characterization of the interface optics in the sample arm}

A set of measurements are presented aimed at characterizing the focusing capabilities of the GF/MS-OCT instrument. A metallic mirror was used as sample and the DC electrical signal from one of the BPD monitor outputs was used to evaluate the confocal profile of the interface optics. The translation stage mTS was moved to 5 positions $\left(\zeta_{1}, \zeta_{2} \ldots \zeta_{5}\right)$ by shifting the lens $\mathrm{L}_{1}-1.5 \mathrm{~mm},-0.75 \mathrm{~mm}, 0 \mathrm{~mm}, 0.75 \mathrm{~mm}$, and $1.5 \mathrm{~mm}$ away from the position for which the beam of light is collimated after $\mathrm{L}_{1}$ (position $\zeta_{3}$ ). Shifting the mTS over a range ( $\delta \zeta$ $=3 \mathrm{~mm}$ ), allows a focus adjustment at the sample position within an approximately $\delta \mathrm{z}=1.2$ mm interval [Fig. 3(a)].

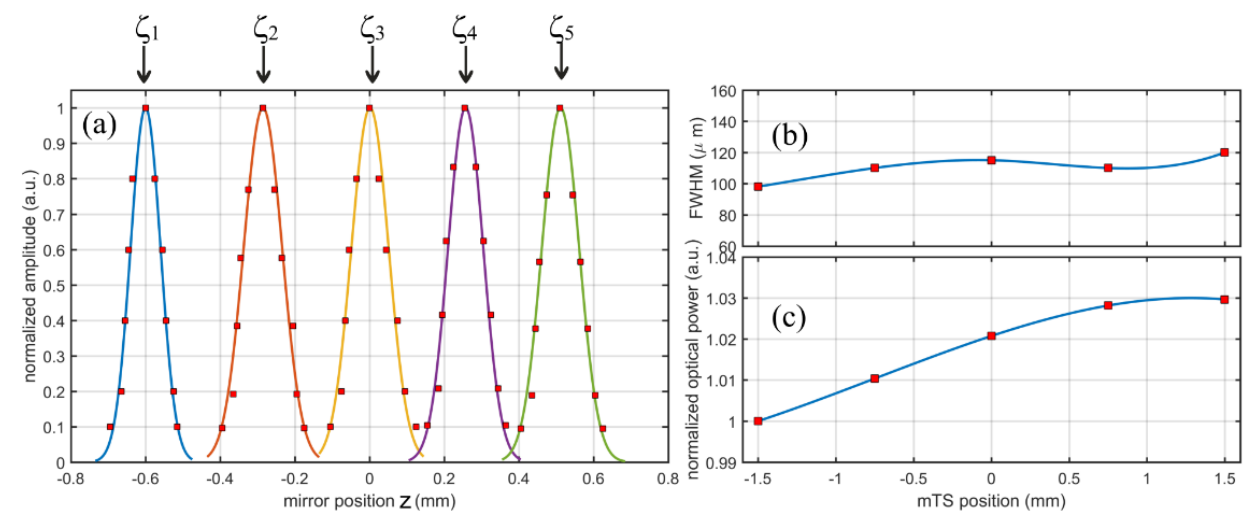

Fig. 3. (a) Normalized confocal profiles obtained for five positions of the lens $L_{1}\left(\zeta_{1}\right.$ to $\left.\zeta_{5}\right)$ over a range of $3 \mathrm{~mm}$. (b) FWHM of the confocal profiles versus position of $\mathrm{L}_{1}$. (c) Normalized optical power after $\mathrm{L}_{1}$ (normalization done with respect to the power measured when $\mathrm{L}_{1}$ is in position $\zeta_{1}$. 
The FWHM values of the confocal profiles shown in Fig. 3(a) were evaluated for each position of the miniature translation stage and are presented in Fig. 3(b). The normalized optical powers measured in the sample arm after $L_{2}$ for the five positions of the miniature stage, $\zeta_{1}$ to $\zeta_{5}$ are presented in Fig. 3(c). A loss of less than 3\% of optical power occurs when the mTS is moved along the optical axis over $3 \mathrm{~mm}$. Because the measurements were done after the galvo-scanning head, the loss may be due to the limited size of galvo-scanner mirrors. With the axial range of $1.4 \mathrm{~mm}$ [Fig. 3(a)], and considering the DOF as given by the FWHM of the confocal profiles in the same Fig. 3(a), we obtain $\mathrm{R}=\mathrm{AR} / \mathrm{DOF} \sim 14$. Consequently, $\mathrm{R}=14$ data acquisitions would be needed, for 14 different positions of $\mathrm{L}_{1}$. However, in practice, as shown below, when imaging scattering samples, the confocal gate profile widens with depth and less repetitions $\mathrm{R}$ are needed.

To demonstrate the capabilities of the focus adjustment procedure presented above, when the DOF is much narrower than the AR, a multi-layer phantom made of 15 microscope coverslips $(22 \mathrm{~mm}$ diameter $\times 0.16-0.19 \mu \mathrm{m}$ thickness) was imaged. In Fig. 4, B-scan OCT images of the phantom obtained by placing the mTS in six positions along the $\zeta$ coordinate, covering a range of $2.6 \mathrm{~mm}$ are demonstrated. The phantom was kept in the same position with respect to $\mathrm{L}_{2}$ in all cases.

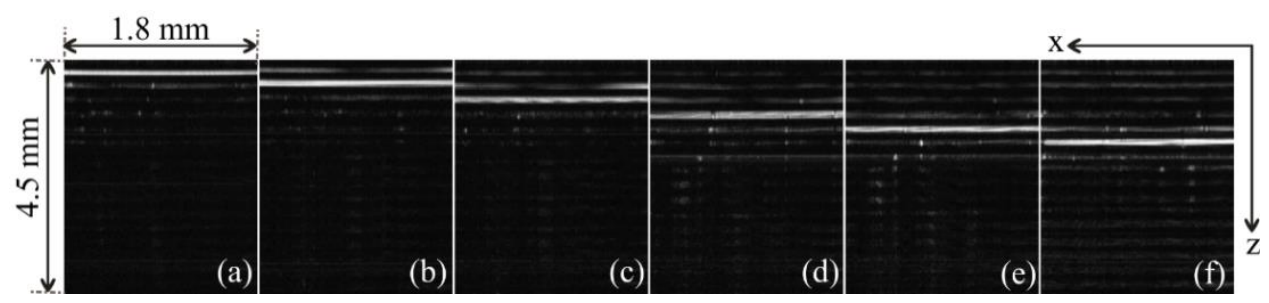

Fig. 4. Six B-scan OCT images collected from the multi-layer phantom using lens $\mathrm{L}_{1}$ in six positions along $\zeta$ covering a range of $2.6 \mathrm{~mm}$. The focus positions in the six frames are axially separated by $0.458 \mathrm{~mm}$, measured in air.

As seen in Fig. 4, the focus moves in depth and an enlargement of the confocal gate profile is also noticed with depth. While in Fig. 4(a), the DOF is restricted to $0.1 \mathrm{~mm}$ as shown in Fig. 3, in Fig. 4(f) all other interfaces within $4.5 \mathrm{~mm}$ become visible. This is attributed to distortion of the wave-front by the intermediate interfaces. To experimentally measure the transversal resolution achievable with the present instrument, a USAF test target was imaged. The resolutions were measured for various positions of the mTS, in each case adjusting the test target axially for maximum brightness. In all the cases, we could measure a transversal resolution of approximately $2.2 \mu \mathrm{m}$.

\subsection{Methods}

\subsubsection{Complex master slave interferometry}

For this study, the complex master slave (CMS) protocol is employed [19]. This relaxes the need to experimentally record channeled spectra for all OPD values needed, as in the first MS reports [15-17]. Only a reduced number of experimental channeled spectra $P$ are required to calibrate the instrument at the Master stage, that are then used to generate a much larger number $\mathrm{Q}$ of masks to be used for the multiple comparison process.

1. With a strong reflector as object, $\mathrm{P}=2$ or more experimental channeled spectra corresponding to different axial positions are stored. This step is performed only once for a given experimental set-up, before data acquisition from the sample to be investigated. For the present set-up, the modification of the OPD was achieved by axially translating $L_{1}$ together with the input fiber. 
2. The $P$ experimental channeled spectra recorded at the step above are then used to theoretically infer the required number $\mathrm{Q}$ of complex masks (using the algorithm presented in [19]). This step establishes the $\mathrm{Q}$ number of depths in the OCT volume investigated and the sampling resolution. If a different axial range with a different sampling is needed, then this process can easily be repeated. This is allowed by the CMS principle, but not possible with the FFT based conventional approach. For the results presented here, $\mathrm{P}=4$ and $\mathrm{Q}=600$.

3. The sample replaces the mirror and $\mathrm{Q}$ comparators produce $\mathrm{Q}$ complex reflectivities for $\mathrm{Q}$ depths from within the sample. En-face and cross-sectional images are produced via matrix multiplications $[17,18]$. This step proceeds as soon as all raw 3D data set has been acquired. For this study, images of lateral size $200 \times 200$ pixels are used, i.e. the 3D data set consists of 40,000 sweeps.

The Master/Slave method, presented in prior reports [15-19] presents several advantages over the conventional FFT based method: (i) direct production of en-face OCT images (no need to render the en-face view from the 3D image), (ii) no need for any resampling/linearization of data (as no FFT is employed) and (iii) tolerance to dispersion mismatch in the interferometer [21].

A separate LabVIEW program is used to drive the two galvo-scanners and create a display with three categories of images. The first category is $\mathrm{M}$ number of en-face OCT images, where $\mathrm{M}$ can be chosen between 1 and $\mathrm{Q}$. For this study, $\mathrm{M}=9$, with images labeled as (e1, e2...e9). An average of the $\mathrm{M}$ images is displayed in the bottom left corner of the refreshing frame as a compound image (c), useful for guidance, as a second type of image. The 3rd type is two B-scan OCT images ( $B_{\mathrm{h}}$ and $B_{\mathrm{v}}$ in the following images) that are cross section images from the sample along the two orthogonal lines shown over the compound image.

For en-face images of 200x200 pixels targeted, at a sweeping rate of $50 \mathrm{kHz}$, a minimum acquisition time of $0.8 \mathrm{~s}$ is required. This requires ramps of $4 \mathrm{~ms}$ applied to the horizontal (line) scanner. We decided to use a single ramp of a triangularly generated shape applied to the line scanner, which determined a line rate of $125 \mathrm{~Hz}$. A saw-tooth signal with a period of $1.6 \mathrm{~s}$ was applied to the frame scanner. In total, 12 images are displayed at a MS frame rate of $0.625 \mathrm{~Hz}$, i.e. every $1.6 \mathrm{~s}$.

\subsubsection{Gabor filtering via the complex master slave method}

The mTS is used to position the focus in the sample at different depths. For each such position, $\mathrm{Q}=600$ en-face OCT images are produced and for guidance in orienting the sample, 12 images are displayed. The $\mathrm{Q}$ images can be used to produce volumes.

In comparison with conventional FFT based OCT technology, where volumes are assembled from many A-scans, here volumes are assembled from en-face OCT images. This difference is also reflected into the implementation of the Gabor filtering. Because the FFT based technology delivers the whole A-scan for each FFT step, for each focus adjustment, the brightest parts of such A-scans are selected from each A-scans while the rests are discarded. The selected parts of A-scans are then stitched together to form a final volume. In our implementation, there is no need to cut and stitch A-scans. Simply, because there is a processor for each depth, we only calculate results for the depths within the confocal gate interval (DOF). Only the en-face images of largest brightness are worked out for each focus position. Then these en-face images are assembled into the final volume. 


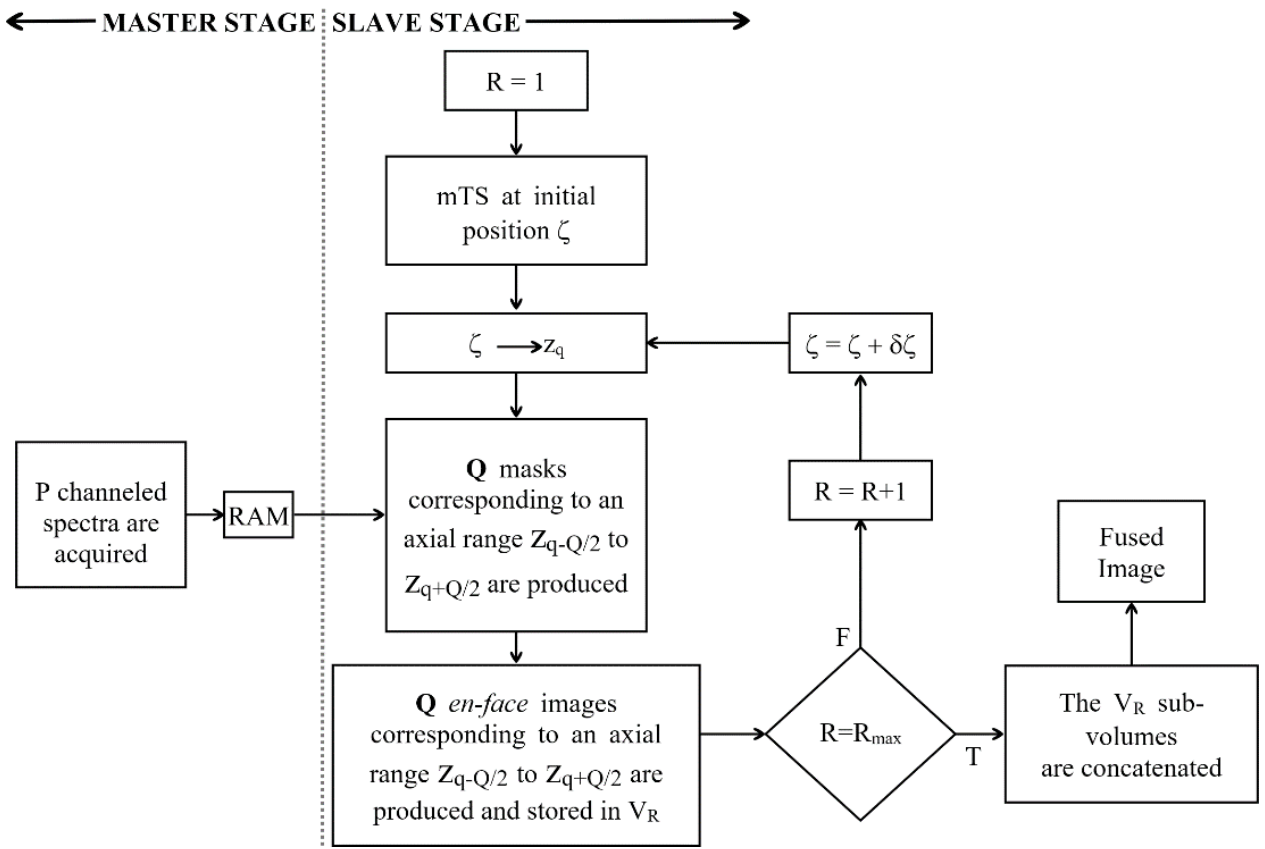

Fig. 5. Flowchart showing the process of producing a Gabor fused image in a master/slave OCT imaging instrument.

In Fig. 5, a flowchart showing the process of producing a fused image using the MS method is shown. The Master stage only involves the recording of $\mathrm{P}$ channeled spectra using a mirror, followed by their storage on the drive of the computer (RAM). At the slave stage, for each focus position, the $\mathrm{P}$ experimentally collected channeled spectra are used to generate $\mathrm{Q}$ masks covering the DOF axial interval centered around the focus position. Let us consider that a number of $R_{\max }$ sub-volumes have to be used to produce the final fused image corresponding to $R_{\max }$ focus positions determined by the position of the mTS. Each position $\zeta$ of mTS, corresponds to a focus position $\mathrm{z}_{\zeta}$ in the image, hence to a certain indexing position $\mathrm{q}$, where $\mathrm{q}$ $=1 \ldots \mathrm{R}_{\max } \times \mathrm{Q}$. Once the axial position of the focus is established, a number of $\mathrm{Q}$ masks are generated and $\mathrm{Q}$ en-face images are produced. Sub-volumes $\mathrm{V}_{\mathrm{R}}$ are generated for each focus position and stored. Finally, when $\mathrm{R}=\mathrm{R}_{\max }$, the $\mathrm{R}_{\max }$ sub-volumes are concatenated into a final fused volume.

\section{Results}

First, we illustrate the focus effect, on the MS global display of the three categories of images produced by the MS protocol. To this goal, a phantom was constructed using 3 microscope coverslips ( $22 \mathrm{~mm}$ diameter $\times 0.16-0.19 \mu \mathrm{m}$ thickness), slightly separated spatially by using thin strips of paper between them, placed by their edges, outside the areas imaged. Figure 6 presents the 12 images frame of the phantom by placing the lens $L_{1}$ in 3 positions along the $\mathrm{z}$ coordinate. For this object, the $\mathrm{Q}=600$ masks (axial points) were aimed to cover a range of $2.7 \mathrm{~mm}$ in air in the final fused image. The image size of each en-face image is $0.5 \times 0.5 \mathrm{~mm}^{2}$, while the size of the B-scan images is $0.5 \mathrm{~mm}$ (lateral) $\times 2.7 \mathrm{~mm}$ (axially). The differential axial distance between the en-face images (e1 - e9) can be chosen during the display and was fixed at $50 \mu \mathrm{m}$. The 9 en-face images are selected between the red (green) lines placed over the two B-scan images. The differential axial distance scales automatically by moving the two red (green) lines. 


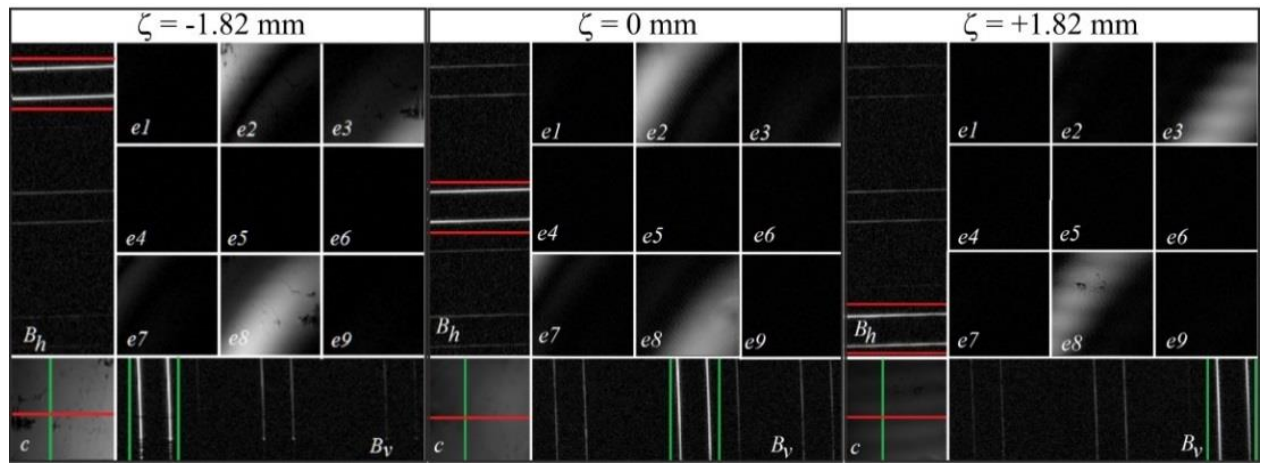

Fig. 6. En-face OCT images (e1 - e9) of a three-layered phantom, separated by $50 \mu \mathrm{m}$, a compound image (c) used for guidance as well as two orthogonal MS based B-scan images $\left(B_{h}\right.$ and $\mathrm{B}_{\mathrm{v}}$ ) used for depth localization of the en-face planes. The three sets of images were obtained for three positions of the $\mathrm{mTS}(\zeta=-1.82,0,+1.82 \mathrm{~mm})$.

3D representations of the 3 volumes of 200 en-face images each are presented in Figs. $7(\mathrm{a})-7(\mathrm{c})$. Each pixel corresponds axially to $\sim 4.5 \mu \mathrm{m}$, hence each sub-volume has an approximate axial length (measured in air) of $\delta z=900 \mu \mathrm{m}$. As the axial position at which the sub-volumes were placed is well determined by the generated masks, the final fused volume is straightforward to produce [Fig. 7(d)].

The experiments presented in Figs. 6 and 7 illustrate good axial confocal gating for specular targets. The phantom used presented little scattering and introduced little aberrations, allowing good axial selection even for the deeper focus.

The combined GF/MS-OCT instrument was then used to image two samples that present more scattering and aberrations for the propagating beam, a piece of cucumber and a plastic encapsulated scorpion. Starting with an axial range of the B-scan image of $5 \mathrm{~mm}$, after localizing the area of interest, the axial range was reduced to $2 \mathrm{~mm}$ (measured in air) by reducing the differential axial range between the masks.

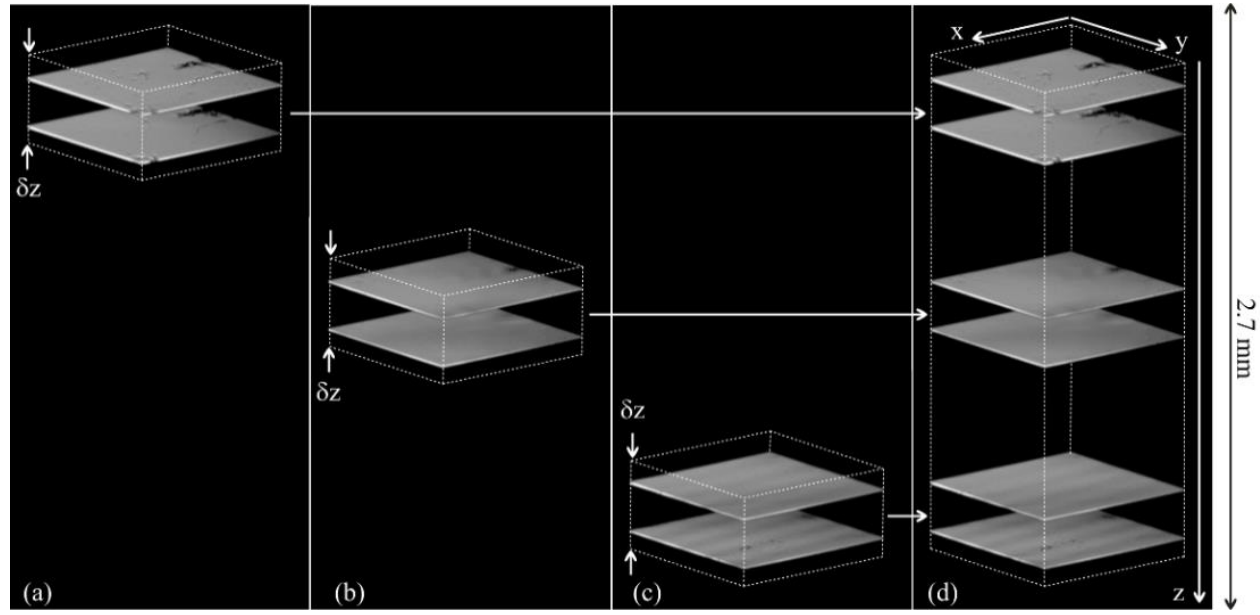

Fig. 7. (a-c) 3D images of the three-slide phantom, built for 3 focus positions. (d) Fused high resolution Gabor-volume obtained by merging 200 en-face images from the first volume using the masks $\mathrm{q}=1$ to 200,200 en-face images from the second volume, using the masks $\mathrm{q}=201$ 400 and 200 en-face images from the 3 rd volume, fusing the masks $q=401$ to 600 . Fused image size $0.75 \times 0.75 \times 2.7 \mathrm{~mm}^{3}$. 

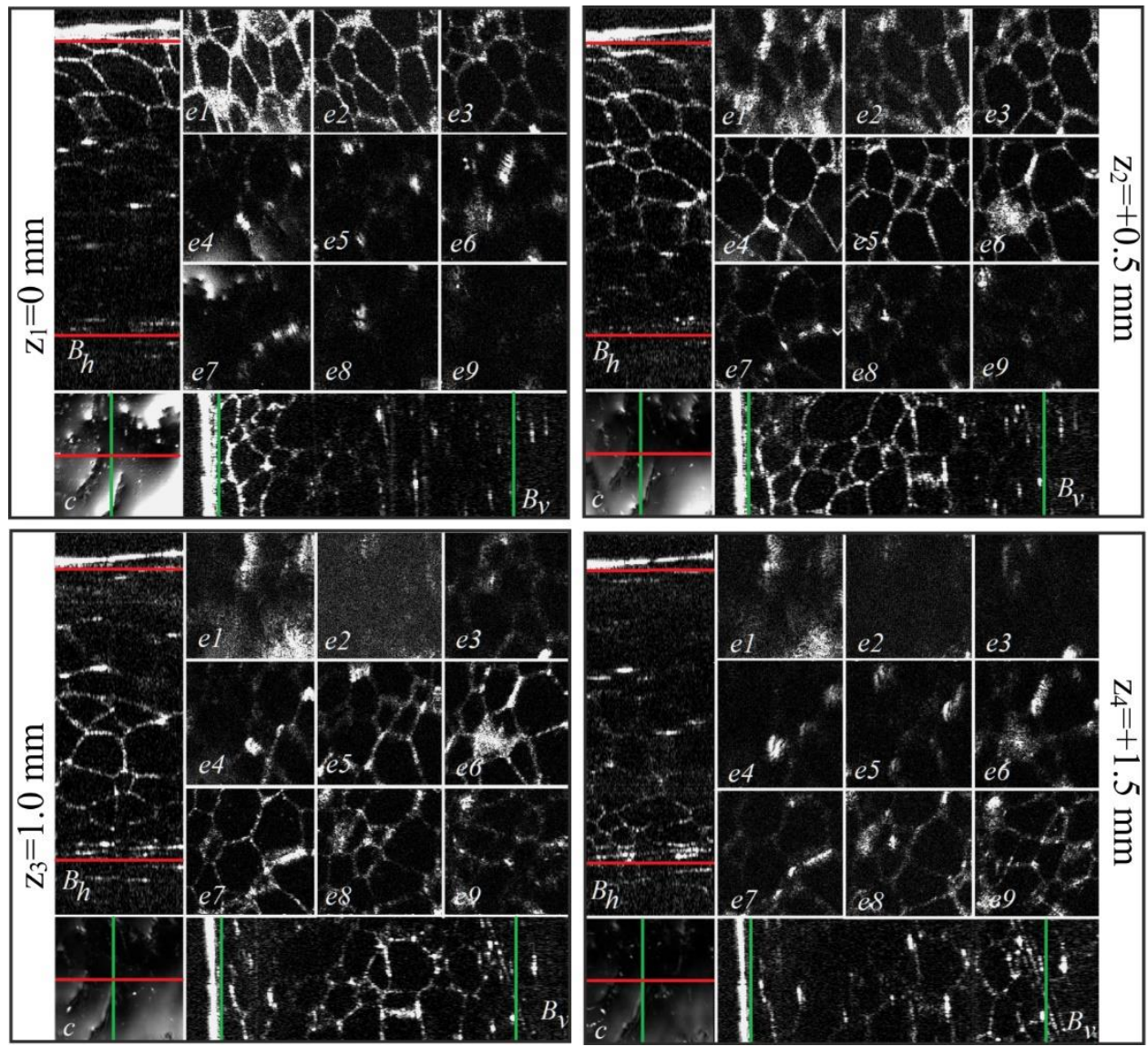

Fig. 8. MS display for 4 focus positions of lens $L_{1}$, for an axial focus shift between frames of $0.5 \mathrm{~mm}$. The MS display consists in 9 en-face OCT images $(e 1-e 9)$ collected from a piece of cucumber, separated axially by $170 \mu \mathrm{m}$ (measured in air), compound image (c) as well as two orthogonal B-scan OCT images $\left(B_{\mathrm{h}}\right.$ and $\left.B_{\mathrm{v}}\right)$. The size of the en-face images is $0.56 \times 0.56 \mathrm{~mm}^{2}$, while the size of the B-scans is $0.56 \mathrm{~mm}$ (lateral) $\times 2 \mathrm{~mm}$ (axially).

Figure 8 presents 9 en-face OCT images collected from a cucumber piece $(e 1-e 9)$, separated axially by $170 \mu \mathrm{m}$ (measured in air), the compound image (c) used for guidance as well as two orthogonal B-scan OCT images $\left(B_{\mathrm{h}}\right.$ and $\left.B_{\mathrm{v}}\right)$, obtained by placing lens $\mathrm{L}_{1}$ in 4 positions covered by the mTS range of $1.5 \mathrm{~mm}$. The size of the en-face images is $0.56 \times 0.56$ $\mathrm{mm}^{2}$, while the size of the B-scans is $0.56 \mathrm{~mm}$ (lateral) $\times 2 \mathrm{~mm}$ (depth). The focus change can be noticed based on the shift of maximum brightness from $e 1, e 2, e 3$ in (a), to seeing better the images in $e 4, e 5, e 6$ in (b), and then to $e 6, e 7, e 8$, in (c) and $e 8, e 9$ in (d).

$3 \mathrm{D}$ representations of these results are presented in Fig. 9. We have left here all volumes, for all depths to allow seeing the difference in brightness and transversal resolution within each volume. However, the same operation as above can be performed, allowed by the MS protocol, by engaging a reduced set of 200 masks only. As commented above in connection with Fig. 7, it can be noticed on the B-scans how the confocal gate width extends with depth, being wider in the set for $\zeta_{4}$ than in the set for $\zeta_{1}=0$. As the DOF looks larger than a quarter of the axial range AR used for this sample, $\mathrm{R}$ was limited to only 4 . The fused set in the last column exhibits not only more uniform brightness along the depth, but the transversal resolution is almost constant along the depth. 


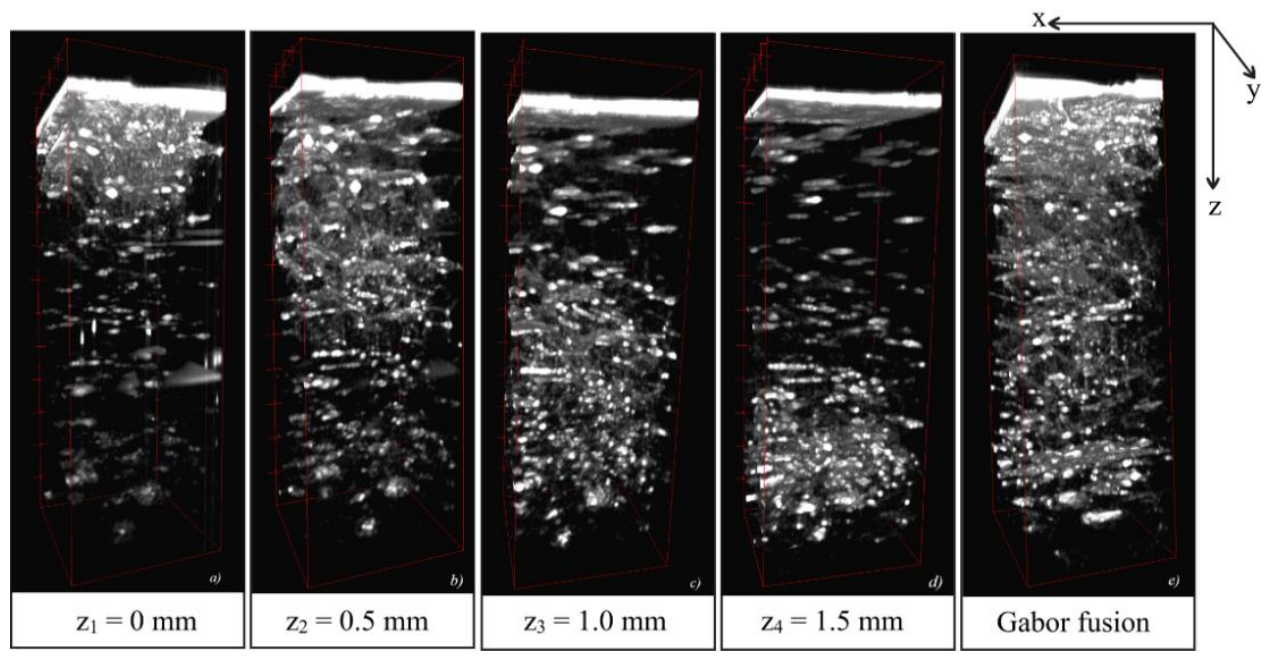

Fig. 9. Cucumber 3D volumes obtained for 4 positions of the mTS (a-d). (d) Gabor-fused volume of 200 depths from each set. Image size $0.56 \times 0.56 \times 2.0 \mathrm{~mm}^{3}$.

We now illustrate the capabilities of the GF/MS-OCT instrument to image a scattering sample placed deep inside a transparent plastic block, as provided in the Issue 1 of the "RealLife Bugs \& Insects", magazine (National Geographic). A scorpion, Manchurian type, found in north east China, is shown in the photograph presented in Fig. 10.
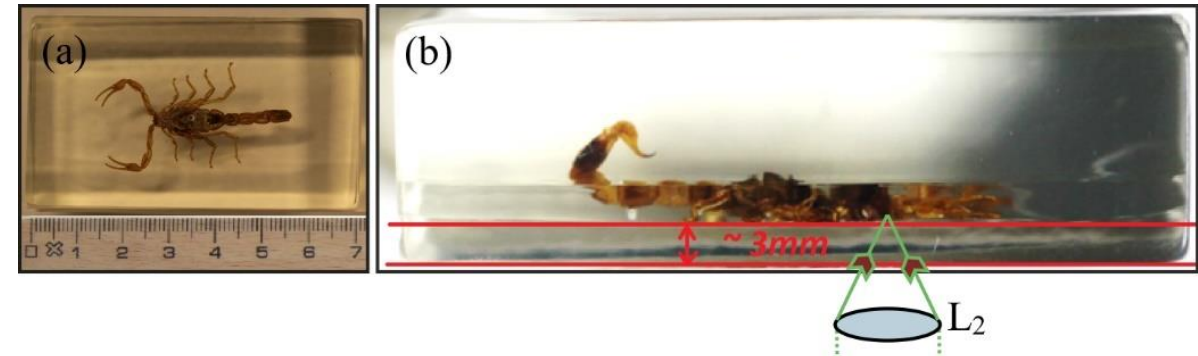

Fig. 10. Photograph of the scorpion embedded in a plastic rectangle. (a) Dorsal view. (b) Side view: the sample was positioned and imaged with its ventral side towards the lens $\mathrm{L}_{2}$. There is about $3 \mathrm{~mm}$ of plastic from the interface air/plastic to the sample.

Here we employ another capability of the MS method, tolerance to dispersion [21]. Because the sample to be imaged is $3 \mathrm{~mm}$ inside the plastic block measured from the surface, the dispersion left unbalanced by the latest can lead to a wider coherence gate than expected. To solve this issue, at the Master stage, the experimental channeled spectra are acquired using a slab of the same plastic material as that used to embed the scorpion. To illustrate the improvement in axial resolution brought by using correct masks, the following two sets of experiments were performed:

(i) At the Master stage, $\mathrm{P}=4$ channeled spectra are acquired using a mirror. The $\mathrm{P}=4$ channeled spectra are used to produce $\mathrm{Q}=1200$ masks, we refer to these as Masks in air, or uncorrected masks. Then these are employed to produce axial reflectivity profiles from the mirror used as object at the slave stage, as well as from the mirror with a $3 \mathrm{~mm}$ plastic slab above, as shown in Fig. 11(a).

(ii) At the Master stage, $\mathrm{P}=4$ channeled spectra are acquired with the $3 \mathrm{~mm}$ slab of plastic material in the reference arm in front of a mirror. The $\mathrm{P}=4$ channeled spectra are used to produce $\mathrm{Q}=1200$ masks. We refer to these as Dispersion affected masks. Then, these 
masks are employed to produce axial reflectivity profiles from the mirror used as object at the Slave stage, as well as from the mirror with the plastic slab above, as shown in Fig. 11(b).
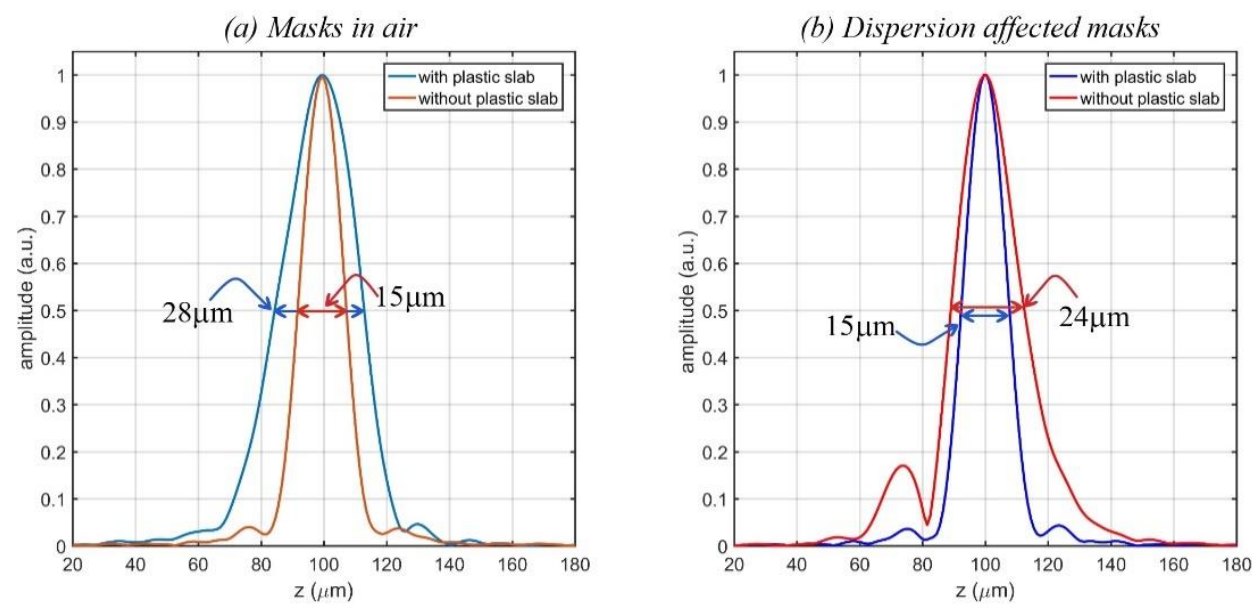

Fig. 11. Axial reflectivity profiles with a plastic slab inserted in the sample arm or not. Left: system equipped with the Masks in air. Right: system equipped with the Dispersion affected masks.

The results presented in Fig. 11 demonstrate that an axial resolution of $15 \mu \mathrm{m}$ is obtained (limited by the bandwidth of the swept source) when the correct set of masks is used for each case: Masks in air in Fig. 11(a) and Dispersion affected masks in Fig. 11(b). When the other set of masks is used, the axial resolution deteriorates to $\sim 24-28 \mu \mathrm{m}$.

Then, when imaging the scorpion, the Dispersion affected masks are used. A similar MS frame to that in Fig. 6 and Fig. 8 is obtained. For this case, in Fig. 12, we show for illustration 6 en-face OCT images of the scorpion, axially separated by $60 \mu \mathrm{m}$ (measured in air), for two different focus positions. The backbone and the rib cage are clearly visible. The size of the enface images is $2.1 \times 2.1 \mathrm{~mm}^{2}$. In the top row, the images are not as bright as the ones in the bottom row and show a poorer transversal resolution as the beam is focused at around $1.0 \mathrm{~mm}$ from $\mathrm{OPD}=0$. For the bottom row, as the focus is placed deeper, at around $1.8 \mathrm{~mm}$ from OD $=0$, sharper brighter features can be observed.

Based on $\mathrm{Q}=600$ en-face $\mathrm{OCT}$ images produced for two positions of the mTS, and using the 600 en-face images obtained after fusion, Visualization 1 was created. There, we are showing, side by side, from the top to the bottom of the sample, en-face images corresponding to the two axial positions of the mTS (left and middle) as well as the en-face in the fused volume (right). The focus was placed towards the top of the image in the left set and towards the bottom in the middle set. In this way, at shallow depths, the resolution/brightness is better in the left set than in the middle, while the other way around at larger axial positions. However, the fused volume exhibits the best resolution and brightness over the whole axial range. 


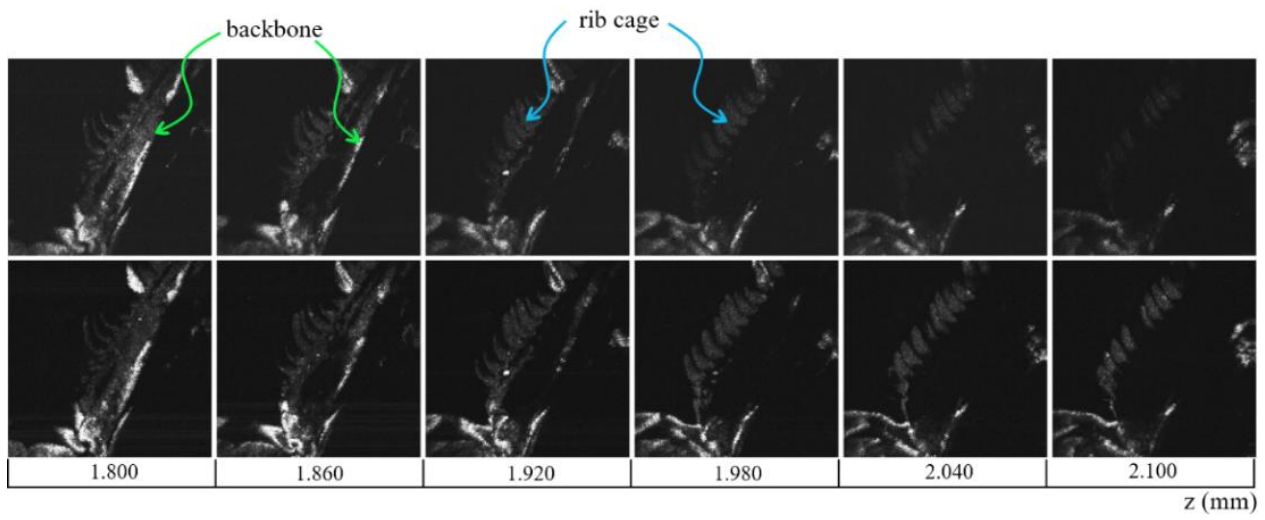

Fig. 12. Sets of 6 en-face images (out of the 9 in the frame) axially separated by $60 \mu \mathrm{m}$. The top row shows lower resolution and brightness images than the images in the bottom row as the focus has been placed at $1 \mathrm{~mm}$ from $\mathrm{OPD}=0$ (top row). For the bottom row, the focus was placed at $\mathrm{z}=1.8 \mathrm{~mm}$.

\subsection{Processing time}

The Gabor fusion method is extremely time consuming as volumetric images must be created for each focusing position before merging the volumes hence created. If the traditional FFT based OCT method is used, for each position of the focusing beam, large volumes in terms of their axial range need to be created where each A-scan is the result of a succession of sequential mathematical operations (apodization, zero padding, dispersion compensation algorithms) and finally of the FFT. As only data collected from within the confocal gate is useful for the final image, the FFT based method produces a large amount of discarded data. The MS technique on the other side, resolves this inconvenience, as only data from within the coherence gate is processed, hence smaller sub-volumes to be fused at the end of the process of constructing the entire image.

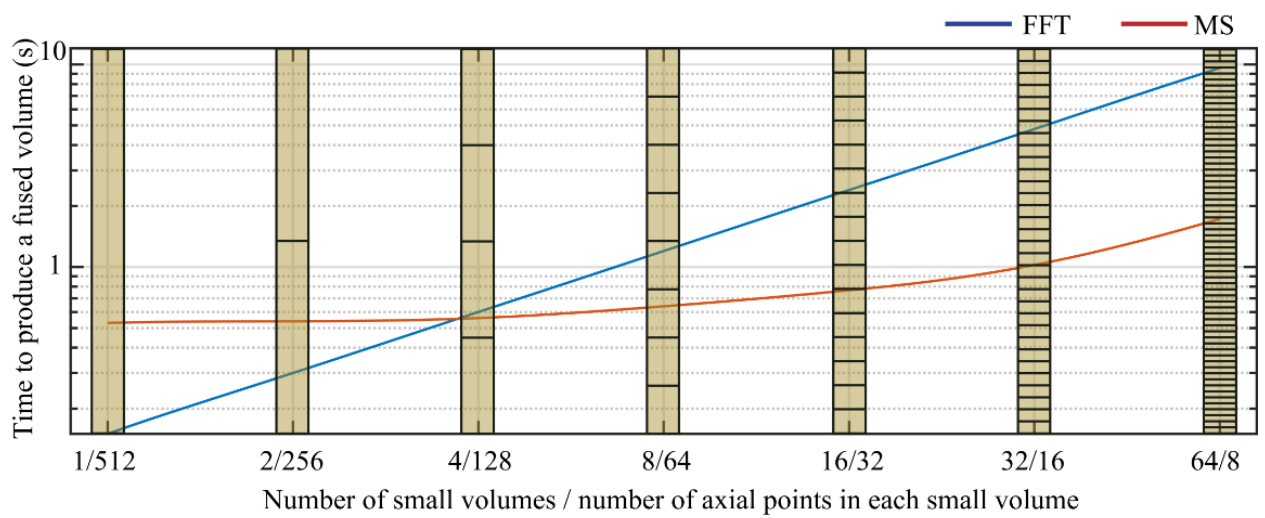

Fig. 13. Time to produce a fused 3D image using the FFT (blue curve) and using the MS method (red curve), for 7 situations, where the final volume has been obtained by concatenating $\mathrm{R}=1,2,4,8,16,32$ and 64 smaller sub-volumes respectively out of axial points in the FFT = Q in the MS case. Data are presented in logarithmic scale.

To evaluate the performance of the MS techniques when used together with the Gabor fusing method, we compared the time to produce a fused 3D image using the MS method with the time required to produce the same image via fast Fourier transform (FFT)s, when data was not resampled or compensated for unbalanced dispersion. To perform the benchmarking, a LabVIEW 2015 (National Instruments, Austin Texas) project was created to run on a computer equipped with an Intel I7-5960X @ 3.0 GHz octacore processor (2 logical cores per 
physical core) and $16 \mathrm{~GB}$ of RAM. Our findings are summarized in Fig. 13.7 situations were considered, in which the final volume has been built from $\mathrm{R}=1,2,4,8,16,32$ and 64 smaller sub-volumes respectively. As we considered that the channeled spectra were digitized using 1024 sampling points, each A-scan in the final volume will contain 512 axial pixels and the MS method uses 512 masks. When 2 sub-volumes are used (two focus positions), each subvolume has 256 axial pixels and the MS uses 256 masks, when 4 sub-volumes are used (four focusing points), each sub-volume has 128 axial pixels and the MS uses 128 masks and so on. In all these calculations, the spectra were still sampled at 1024 spectral points. The time to produce a sub-volume using the FFT implementation of OCT is the same irrespective of the number of axial pixels in the sub-volume, so it is expected that the time to produce the final $3 \mathrm{D}$ volume using the FFT method to be proportional with the number of sub-volumes. When using the MS method, the smaller the number $\mathrm{Q}$ of axial points, less is the computation time. As illustrated in Fig. 13, by using a number of sub-volumes R > 4, with the MS method, the process of producing Gabor fused volumes is shorter than using the FFT method. This can be simply understood, as the number of axial points scales down with R. By increasing R, the overall calculation time is not affected, as the same number of masks are used irrespective of $\mathrm{R}$, per the final volume. However in practice, due to the limitation of cores in the PC used, we noticed a slight increase in the time required when increasing R. If GPUs are used, this may not be a problem at all.

For the data presented in Fig. 13, the fused volume has a size of $200 \times 200 \times 512$ pixels $^{3}$. The time to produce any sub-volume using FFT is $\sim 0.15 \mathrm{~s}$, which as shown by the blue graph would give $\sim 4 \mathrm{~s}$ for $\mathrm{R}=32$, while the MS method would require $\sim 1 \mathrm{~s}$, as shown by the red graph.

It is important to note that, for our particular case, if more than 4 focusing positions are required for fusing the volume, which is the case in most practical cases, the MS method becomes faster than the FFT based method. In case data needs to be resampled, or dispersion compensation is needed, the FFT method is even slower. As mentioned above, the MS method does not need resampling nor dispersion compensation of the system [15-19, 21].

\section{Discussion and conclusions}

In this study, Gabor filtering is combined with MS-OCT principle to provide constant transversal resolution from the sample investigated. Different modalities have been used to shift the focus for Gabor filtering, such as an electrical lens [12,22] or a deformable mirror [23]. Here, a miniature translation stage is employed to displace a collimating lens. This mechanical solution was mainly chosen to avoid problems of electrical lenses such as aberrations and losses. In addition, there is no need for fast focus change as the system acquires frames at a rate of $1 \mathrm{~s}$ per frame, therefore several milliseconds allocated to focus change can be tolerated. In our case, the time necessary to move the stage carrying the lens from a focus position to the next is only $0.1 \mathrm{~s}$, much less than $6.4 \mathrm{~s}$ required for acquisition of 4 MS frames.

The instrument is characterized in terms of its performance and its capabilities are illustrated with en-face, cross-section and 3D images of various objects. Each MS frame refreshes at $1.6 \mathrm{~s}$ and contains $\mathrm{M}=9$ en-face images, $2 \mathrm{~B}$-scans and a compound image. The time for each MS frame is limited by the speed of the swept source. At this sweeping rate, $\mathrm{CPU}$ processing was sufficient to work out $\mathrm{Q}=600$ en-face images, work out $2 \mathrm{~B}$-scans and to display the 12 images in each MS frame. As shown in Fig. 13, for $\mathrm{R}=4$, the calculation requires $0.6 \mathrm{~s}$. This means that the CPU could still be used for a double sweeping rate, i.e. the same processing is sufficiently fast to cope with the next Axsun swept source in the range, that operates at $100 \mathrm{kHz}$. Using an Axsun swept source at $100 \mathrm{kHz}$, the same protocol allowed a MS frame rate of $0.9 \mathrm{~s}$, leaving some reserve for calculations. Further increase in the sweeping would require GPUs to cope with the reduction in the time taken for acquisition of 
the same number of lateral pixels, in order to produce the same type of the MS frame presented here at the same frame rate (with a delay no more than a frame period).

The use of the Master/Slave method allows evaluation of transversal resolution and of sensitivity variation with depth simultaneously, by providing data from multiple-depth en-face images in real time.

The combination of the Gabor filtering with a Master/Slave based OCT instrument presents several advantages over its combination with a conventional FFT based one:

(i) There is no need for cut and stitch of A-scans.

(ii) Data outside the coherence gate does not need to be discarded, so that calculations are not performed for depths outside the confocal gate, something that it is not possible with the conventional FFT based OCT technology.

(iii) Using the MS procedure, the en-face OCT images can be produced directly. In this way, there is no need to render the en-face views from each volumetric image obtained for each focus positions. In this way, less data is produced than when using FFT. The user has direct access to the en-face views exactly as in TD-OCT while the high sensitivity in the image is still preserved as MS is based on a spectral domain principle.

(iv) As no FFT is employed, there is no need for any resampling/linearization of data, and this leads to time saving. The method presented is also more data processing efficient, as no data is discarded. Consequently, the MS can produce a volumetric image faster than the FFT based technique when data need to be resampled [24]. Even when no resampling is needed, the time required by the MS procedure combined with GF is less than that of FFT based OCT combined with GF, when the number of sub-volumes to be produced is at least $R=4$ (which is a typical case in practice).

(v) As MS is tolerant to dispersion in the interferometer, there is no need to perfectly balance the dispersion, so there is no need for any dispersion compensation procedure.

The MS method illustrated here uses sets of masks covering a limited spectral range, matching the focus position in depth for each adjustment of the confocal gate. Due to this, the method presented is closer to that reported in a recent paper on Gabor filtering [25] than in the original paper [12]. However, in [25], where Gabor filtering is performed in the spectral domain, the processing is still via a FFT. This requires data resampling and correction of dispersion, both intrinsically addressed by the MS method.

\section{Acknowledgments}

R. Cernat, T.G. Heath, P. Keane, R. Rajendram and A. Podoleanu are supported by the NIHR Biomedical Research Centre at Moorfields Eye Hospital NHS Foundation Trust and the UCL Institute of Ophthalmology. A. Podoleanu and A. Bradu also acknowledge by the European Research Council (http://erc.europa.eu), grant 249889. A. Podoleanu is also supported by the Marie Curie EID UBAPHODESA FP7-PEOPLE-2013-ITN 607627 and by the Royal Society Wolfson Research Merit Award. N. M. Israelsen, O. Bang and A. Podoleanu acknowledge support from Innovation Fund Denmark through the Shape OCT grant No. 4107-00011A. A Bradu is also supported by the EPSRC - EP/N019229/1. S. Rivet acknowledges the MarieCurie Intra-European Fellowship for Career Development, No. 625509. R. Cernat and A. Bradu equally contributed to this paper. 